\title{
Chondrosarcoma of Bone
}

\section{Lessons From 46 Operated Cases in a Single Institution}

\author{
Olavo Pires de Camargo MD, PhD, André Mathias Baptista MD, \\ Marcelo Junqueira Atanásio, Daniel Reis Waisberg
}

Published online: 15 May 2010

(C) The Association of Bone and Joint Surgeons $\mathbb{R} 2010$

\begin{abstract}
Background Bone chondrosarcomas are rare malignant tumors that have variable biologic behavior, and their treatment is controversial. For low-grade tumors, there is no consensus on whether intralesional en bloc resections are the best treatment.

Questions/purposes We therefore compared patients with Grade 1 and Grade 2 primary central chondrosarcomas to (1) determine difference in survival and (2) local recurrence rates; and (3) determine any association of histological grade with some clinical and demographic characteristics. Methods We retrospectively reviewed 46 patients with grade 1 and 2 chondrosarcomas. There were 25 men and 21 women with a mean age of 43 years (range, 17-79 years). Minimum followup was 32 months (mean, 99 months; range, 32-312 months) for the patients who remained alive
\end{abstract}

Each author certifies that he or she has no commercial association (eg, consultancies, stock of ownership, equity interest, patent/licensing arrangements, etc) that might pose a conflict of interest in connection with the submitted article.

Each author certifies that his or her institution has approved the human protocol for this investigation, and that all investigations were conducted in conformity with ethical principles of research, and that informed consent for participation in the study was obtained.

O. P. de Camargo $(\bowtie)$

Department of Orthopaedics, University of São Paulo, Rua Ovídio Pires de Campos, 333 - Cerqueira César, São Paulo CEP 05403-010, Brazil

e-mail: olapcama@uol.com.br

A. M. Baptista

Orthopaedic Oncology Group, University of São Paulo, São

Paulo, Brazil

M. J. Atanásio, D. R. Waisberg

University of São Paulo Medical School, São Paulo, Brazil in the end of the study. Twenty-three of the tumors were intracompartmental (Enneking A); of these, 19 were Grade 1 and 4 were Grade 2. Twenty-three tumors were extracompartmental (Enneking B); of these, 4 were Grade 1 and 19 were Grade 2. Twenty-five patients underwent intralesional resection, 18 had wide resection, and three had amputations.

Results The overall survival rate was $94 \%$ and the disease-free survival rate was $90 \%$. Among the 23 Grade 1 tumors, we observed six local recurrences and none of these patients died; among the 23 Grade 2 tumors, 10 recurred and two patients died. Local recurrence negatively influenced survival.

Conclusions For lesions with radiographic characteristics of intracompartmental Grade 1 chondrosarcoma, we believe intralesional resection followed by electrocauterization and cement is the best treatment. When the imaging suggests aggressive (Grade 2 or 3 ) chondrosarcoma, then wide resection is promptly indicated.

Level of Evidence Level IV, case series. See Guidelines for Authors for a complete description of levels of evidence.

\section{Introduction}

Chondrosarcoma is the second most common bone sarcoma [11, 18], accounting for around $20 \%$ of bone sarcomas in the United States [14]. It arises mainly in patients over 40 years of age, has a slight male predominance, and can be either primary or secondary to a previous benign chondral lesion such as an osteochondroma or an enchondroma. Histologic grading, proposed by Lichtenstein and Jaffe [13], is intended to reflect the aggressiveness of the lesion, with Grade 1 tumors designated as low-grade and grades 2 and 3 as high-grade neoplasms. Both musculoskeletal oncologists 
and pathologists generally use plain radiographs in assessing the aggressiveness of the tumor. Nevertheless, there is considerable controversy regarding the low reliability of the pathologic and radiographic grading of these tumors, as demonstrated in the SLICED study [16].

The mainstay of treatment is surgery, once chemotherapy or radiation therapy is not effective [5-7]. Patients with Grade 1 tumors have a 90\% 5-year survival rate, whereas only $40 \%$ to $60 \%$ of patients with grades 2 and 3 tumors will survive after 5 years [7, 8]. A recent population study suggested that patients who survive for ten years are more likely to die of events that were unrelated to chondrosarcoma, and that female sex, a low histological grade, and local surgical stage are associated with a considerable disease-specific survival benefit [10]. However, great variation in biologic behavior between grades is characteristic of chondrosarcoma, ranging from a latent or slow-growing tumor to an aggressive sarcoma, with rapidly developing pulmonary metastasis. Low-grade tumors usually have a more benign behavior, as well as a less aggressive aspect on imaging with no cortical expansion, destruction, or soft tissue mass. High grade chondrosarcomas on the other hand typically exhibit all three.

A number of authors recommend wide resections for grade 2 and 3 tumors and for extracompartmental Grade 1 lesions $[1,3,9,12]$. However, there is some controversy regarding the adequate surgical margin for low-grade intracompartmental chondrosarcomas. Some authors advocate wide resection [8, 11], while others believe intralesional resection augmented with a surgical adjuvant, such as liquid nitrogen, phenol, electrocautery and argonbeam laser, is adequate [7, 17].

Unfortunately, biopsy in chondral intramedullary lesions is unreliable $[8,14]$ for grading and often misleads the surgeon to inadequate surgery [5, 6]. Therefore, clinical history and imaging studies are extremely important when choosing the appropriate surgical technique. Radiographic characteristics such as cortical thickening, expansion or destruction, and soft tissue mass usually point to a more aggressive, high-grade tumor $[8,15]$.

We therefore compared patients with Grade 1 and Grade 2 primary central chondrosarcomas to (1) determine whether there is any difference in survival rates and (2) local recurrence rates; (3) determine whether there is any association of histological grade with clinical and demographic characteristics; and (4) establish a treatment algorithm of central chondral bone lesions on the associations.

\section{Patients and Methods}

We retrospectively reviewed all 94 chondrosarcomas treated from 1986 to 2006, with a minimum followup for the living patients of 30 months. Of these we excluded 48 secondary, mesenchymal, dedifferentiated periosteal and grade 3 chondrosarcomas, which left 46 grade 1 and 2 primary central chondrosarcomas. There were 25 men (54\%) and 21 women (46\%) and the mean age was 46 years (range, 17-79 years). Twenty-three of $46(50 \%)$ were histologic Grade 1 tumors, and, thus, the remaining 23 were Grade 2 cases. The minimum followup was 32 months (mean, 99 months; range, 32-312 months) for the patients who remained alive in the end of the study. Three patients died, at 2, 17 and 43 months of followup (mean considering all patients: 94 months).

We recorded the age, gender, followup period (in months), anatomic location (axial or appendicular skeleton, upper or lower limb), staging according to the Enneking system [4] (intra- [A] or extracompartmental [B]), type of surgery, surgical margins, surgical complications, local recurrence, and survival. The femur was the most common anatomic site, with 21 cases $(46 \%)$, followed by the humerus (nine) and the iliac bone (five). Other sites included proximal phalanx (three), scapula (one), metacarpal (one), lumbar vertebrae (L2) (one), and pubis (one) (Fig. 1). Thirty-nine tumors (85\%) were located in the appendicular skeleton and only seven $(15.2 \%)$ in the axial skeleton. Twenty-five (54\%) were in the lower limbs and $14(46 \%)$ in the upper limbs. We decided to include axial and hand cases in this study due to epidemiological reasons, as this is a very rare tumor. Half the cases were intracompartmental (Enneking stage IA or IIA) and the other half were extracompartmental tumors (Enneking IB, IIB, and IIIB) (Table 1). One case was extracompartmental and metastatic at diagnosis (Enneking IIIB).

Biopsy was performed in 25 of 46 cases (54\%). All slides from the biopsies and resected specimens were reviewed by two pathologists experienced in musculoskeletal oncology and the diagnosis of primary central chondrosarcoma was confirmed. Histologic grading was determined according to Lichtenstein and Jaffe [13], based on the resected specimen. The correspondence between the grade obtained in biopsy and the resected specimen was $60 \%$ (Table 1). Biopsy was performed in 11 Grade 1 cases and good correspondence was found in eight. Among the 14 biopsied Grade 2 cases, correspondence to the resected specimen occurred in seven cases.

Among the 23 Grade 1 cases, there were 15 men and eight women, with a mean age of 43.6 years (SD, 15.3). Nineteen had intracompartmental (IA) and four had extracompartmental (IB) tumors. Twelve tumors were localized in the femur, seven in the humerus, two in the proximal phalanx, one in the metacarpal, and one in the iliac bone. Mean followup in this group was 94.8 months (range, 32-312 months). Nineteen patients underwent intralesional resection, three underwent wide resection, and 


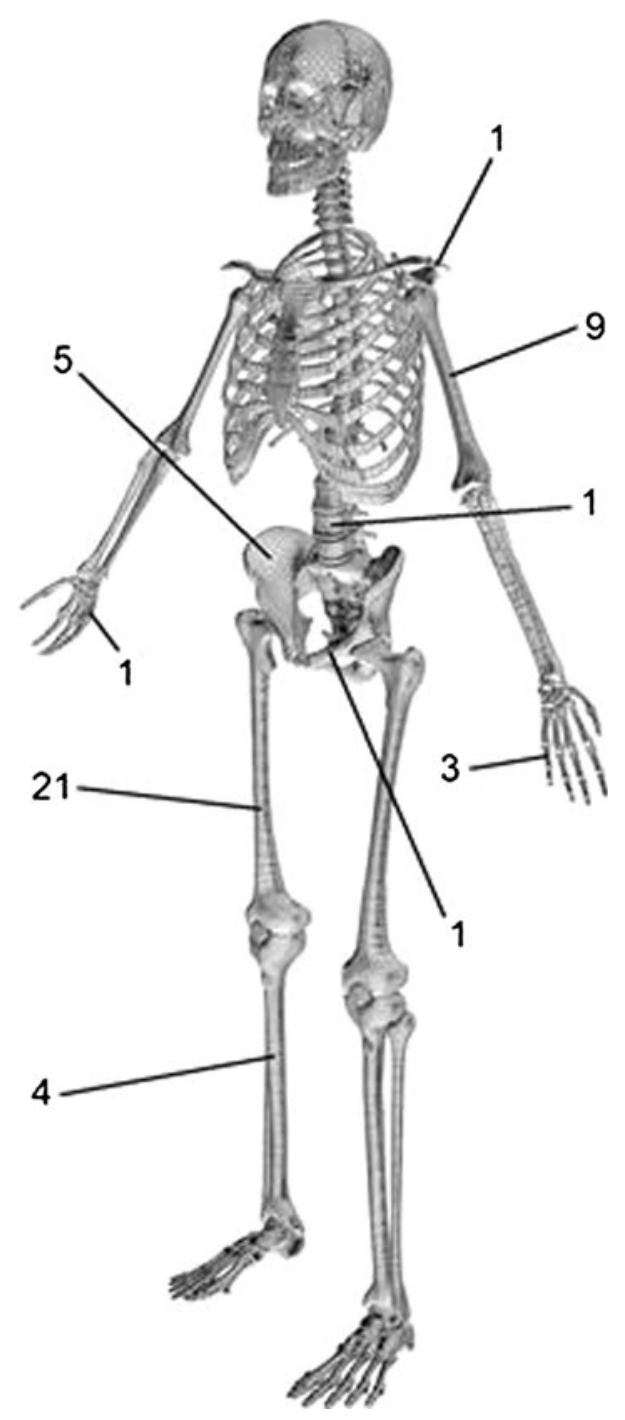

Fig. 1 The illustration shows the frequency distribution of patients according to the anatomic site of the bone chondrosarcomas.

one had an amputation. Among the Grade 2 tumors, 17 were in men and six were in women. The mean age for this group was 42.5 years (SD, 18.3). Eighteen lesions were Stage IIB, four Stage IIA, and one Stage IIIB (pulmonary metastasis). Ten cases were localized in the femur, four in the iliac bone, four in the tibia, two in the humerus, and one each in the scapula, lumbar vertebrae (L2), and pubis. The average followup in this group was 103.9 months (range, 46-207 months). Fifteen patients had wide resection, six had intralesional resection, and two underwent amputation. Although the age was similar in both groups, there were more women with Grade 1 tumors and more men with Grade 2 tumors. Grade 1 lesions were equally distributed between upper and lower limbs. Grade 2 tumors, however, were more common in the lower limbs. We noted a trend towards Grade 1 tumors being appendicular and Grade 2 lesions being located in the axial skeleton. Most Grade 1
Table 1. Frequency distribution of nominal (qualitative) attributes of patients. Evaluation of casuistic according to histologic grading (1 and 2). Comparison of the groups for the chi-square statistic test or the Fisher's exact test ( $\alpha=0.05$, bilateral)

\begin{tabular}{|c|c|c|c|c|}
\hline \multirow[t]{2}{*}{ Attribute } & \multicolumn{2}{|c|}{ Histologic Grade } & \multicolumn{2}{|c|}{ Comparison } \\
\hline & 1 & 2 & Test & $\mathrm{p}$ \\
\hline \multicolumn{5}{|l|}{ Gender } \\
\hline Female & 15 & 6 & $X^{2}$ & $0.008^{*}$ \\
\hline Male & 8 & 17 & & \\
\hline \multicolumn{5}{|c|}{ Mean age (range) } \\
\hline Years & $43.6(18-79)$ & $42.5(17-79)$ & $\begin{array}{c}\text { Student t } \\
\text { test }\end{array}$ & 0.67 \\
\hline \multicolumn{5}{|l|}{ Biopsy } \\
\hline No & 12 & 9 & $X^{2}$ & 0.37 \\
\hline Yes & 11 & 14 & & \\
\hline \multicolumn{5}{|c|}{ Biopsy accuracy } \\
\hline Correct & 8 & 7 & Fisher & 0.41 \\
\hline Incorrect & 3 & 7 & & \\
\hline \multicolumn{5}{|c|}{ Anatomic localization } \\
\hline Axial skeleton & 1 & 6 & Fisher & 0.10 \\
\hline $\begin{array}{l}\text { Appendicular } \\
\text { skeleton }\end{array}$ & 22 & 17 & & \\
\hline \multicolumn{5}{|c|}{ Appendicular skeleton } \\
\hline Lower limb & 11 & 14 & Fisher & $0.049 *$ \\
\hline Upper limb & 11 & 3 & & \\
\hline \multicolumn{5}{|c|}{ Enneking staging } \\
\hline A & 19 & 4 & Fisher & $\sim 0.00 *$ \\
\hline B & 4 & 19 & & \\
\hline \multicolumn{5}{|l|}{ Type of surgery } \\
\hline Intralesional & 19 & 6 & Fisher & 0.08 \\
\hline Wide & 3 & 15 & & \\
\hline \multicolumn{5}{|c|}{ Surgical complications } \\
\hline No & 21 & 17 & Fisher & 0.24 \\
\hline Yes & 2 & 6 & & \\
\hline
\end{tabular}

tumors were intracompartmental, and Grade 2 tumors usually were extracompartmental.

Twenty five patients had intralesional resection and electrocauterization, followed by cementation of the resulting cavity (Table 1). Eighteen patients underwent wide resection and three underwent amputation. Most patients with Grade 1 tumors had intralesional resection and cement, while most with Grade 2 tumors had en bloc resection.

The patients were evaluated each 3 months for the first 2 years, then each 6 months until 5 years and annually afterwards. Local radiographs and physical exam were performed at each evaluation. If local recurrence was suspected, additional imaging was requested (CT scan or MRI). Chemotherapy and radiation were not used. Lung metastasis was assessed with CT scan. Surgical 
margins were evaluated according to the Enneking staging system [4].

The frequency distribution of each attribute (patient characteristics) was presented in summarized contingency tables to describe the nominal data. To examine a possible association between gender and histological grade, the chisquare test was used. This test was also used to verify if the presence of biopsy was associated with the histological grade. Fisher's exact test was used to verify if biopsy accuracy, anatomic localization of the tumor, appendicular skeleton, Enneking staging, type of surgery or surgical complications were associated with histological grade, 1 or 2. The Kaplan-Meier survival was determined for patient survival and disease-free survival according to histologic grade $(1,2)$. To compare the survival between the different groups of patients (according to grade), the Cox-Mantel log-rank test was used.

\section{Results}

The overall survival rate was $94 \%$ (43/46) (Fig. 2). Four patients in the Grade 2 group and 2 in Grade 1 were alive with disease (13\%). Thus, the disease-free survival rate was $89 \%$ (38/46). Local recurrence rate was $26 \%(6 / 23)$ in Grade 1 tumors and $43 \%$ in Grade 2. The recurrences were detected in a mean time of 23.5 months (range, 9-46 months). Among these six local recurrences, one showed as histological Grade 1 and the other five became Grade 2 lesions. These patients then underwent four wide resections and two intralesional resections. Both intralesional procedures and one of the four wide resections recurred for a second time. One patient had an amputation and was free of disease at last followup; two patients were

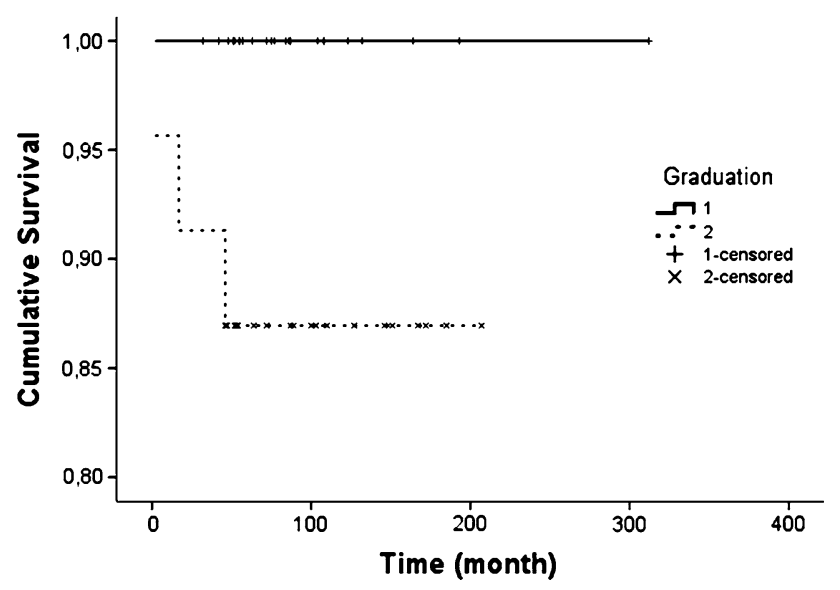

Fig. 2 Kaplan-Meier curves for overall survival (months) according to histologic grade (1, 2 and both). Comparison by the Cox-Mantel log-rank test for trends $(\alpha=0.05$, bilateral). lost to followup. Overall survival rate in Grade 1 tumors was $100 \%$ (Fig. 2), although two patients were alive with disease at the last evaluation, which were at 32 and 87 months of followup. Therefore, the disease-free survival rate was $91.3 \%$ (Fig. 3A-D).

Local recurrence rate for the Grade 2 group was $43.4 \%$ (10/23). Among the 11 recurrences, one patient was inoperable and died of the disease 17 months after diagnosis (recurrence grade was unknown). Eight tumors recurred as histologic Grade 2, one as Grade 1, and one as Grade 3 chondrosarcoma; in all eight patients we reviewed the original histological sections and confirmed they changed grade. Three were treated with en bloc resection, three with intralesional resection, three with amputation (one developed pulmonary metastasis and died of the disease at 46 months), and one with soft tissue resection only. Five patients $(50 \%)$ had second recurrences. Three had been treated with intralesional procedures, one with en bloc resection, and one with soft tissue resection. Among these five patients, two had amputations (one died of brain and pulmonary metastasis 2 months after surgery), one underwent an additional intralesional resection and was free of disease at last followup, one had a third local recurrence, was amputated, and was free of disease, and one who was treated with intralesional resection was lost to followup. The overall survival rate for Grade 2 chondrosarcomas in this study was $91.3 \%$ (Fig. 2). Three patients died of the disease at 2, 17, and 46 months and one was alive with disease at the last followup (64 months), thus establishing the disease-free survival rate at $82.6 \%$ (Fig. $4 \mathrm{~A}-\mathrm{C}$ ). The local recurrence rate was $32 \%$ in intralesional resections and $44.4 \%$ in en bloc resections. Among Grade 1 lesions treated with en bloc resection, there was one recurrence (33\%). On the other hand, there was a $26.3 \%$ local recurrence rate (5/19) with intralesional resections in Grade 1 tumors. In Grade 2 tumors, we had a local recurrence rate of $46.6 \%$ in en bloc resection cases, as opposed to $50 \%$ in intralesional resections.

No association was found between histological grades and clinical or demographical data.

Surgical complications occurred in two Grade 1 cases and six Grade 2 patients (17\% of the total) (Table 1). There were four cases of endoprosthesis loosening, three fractures after intralesional resections and cement, and one case of infection after wide resection and reconstruction with endoprosthesis. We believe that the fractures occurred more due to the lack of prophylactic osteosynthesis, than to the electrocauterization and cementation, although we do not have data to prove it.

Based on the data we constructed an algorithm based on the treatment choices made with these chondrosarcoma patients. This algorithm illustrates the rationale for the bone lesion treatment that is used in our service and the 
Fig. 3A-D These images show grade 1 distal femur chondrosarcoma. (A) Preoperative radiography; (B) coronal T1-weighted magnetic resonance imaging MRI image; (D) postoperative radiography after intralesional resection, electrocauterization, and cement augmented by prophylactic osteosynthesis. (MRI); (C) coronal T2-weighted
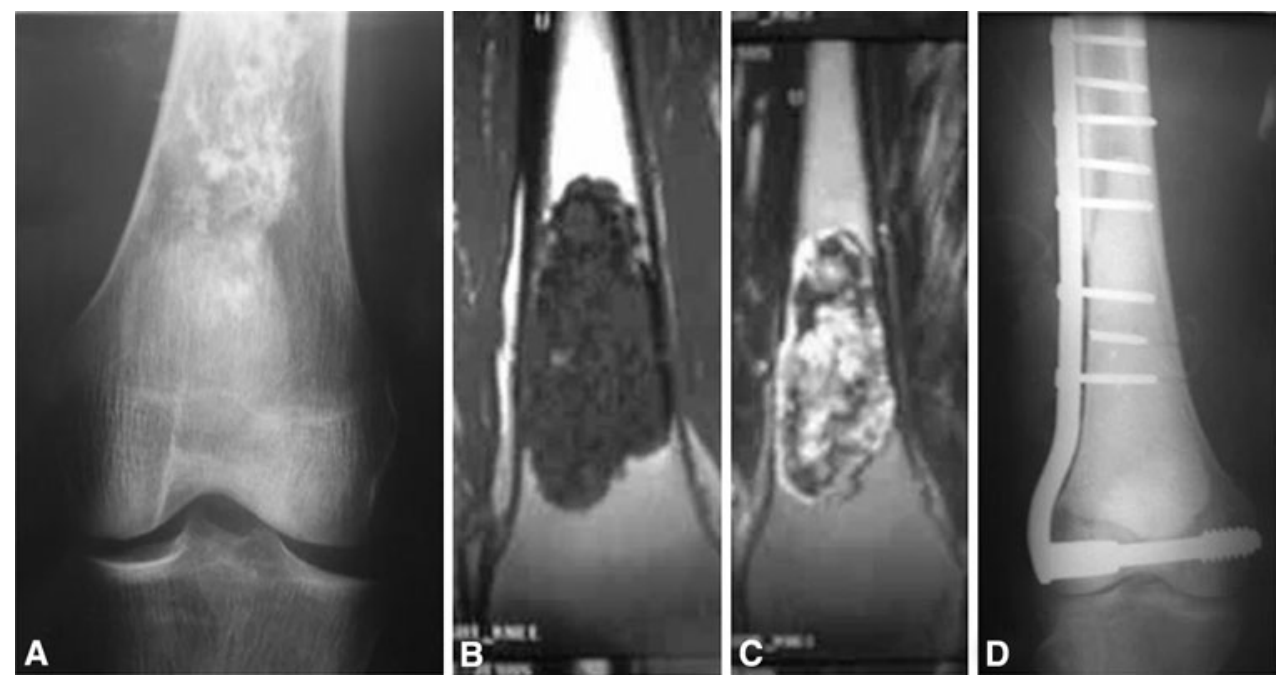

Fig. 4A-C Grade 2 distal femur chondrosarcoma as shown by; (A) preoperative radiography; (B) macroscopic aspect; (C) postoperative radiography.
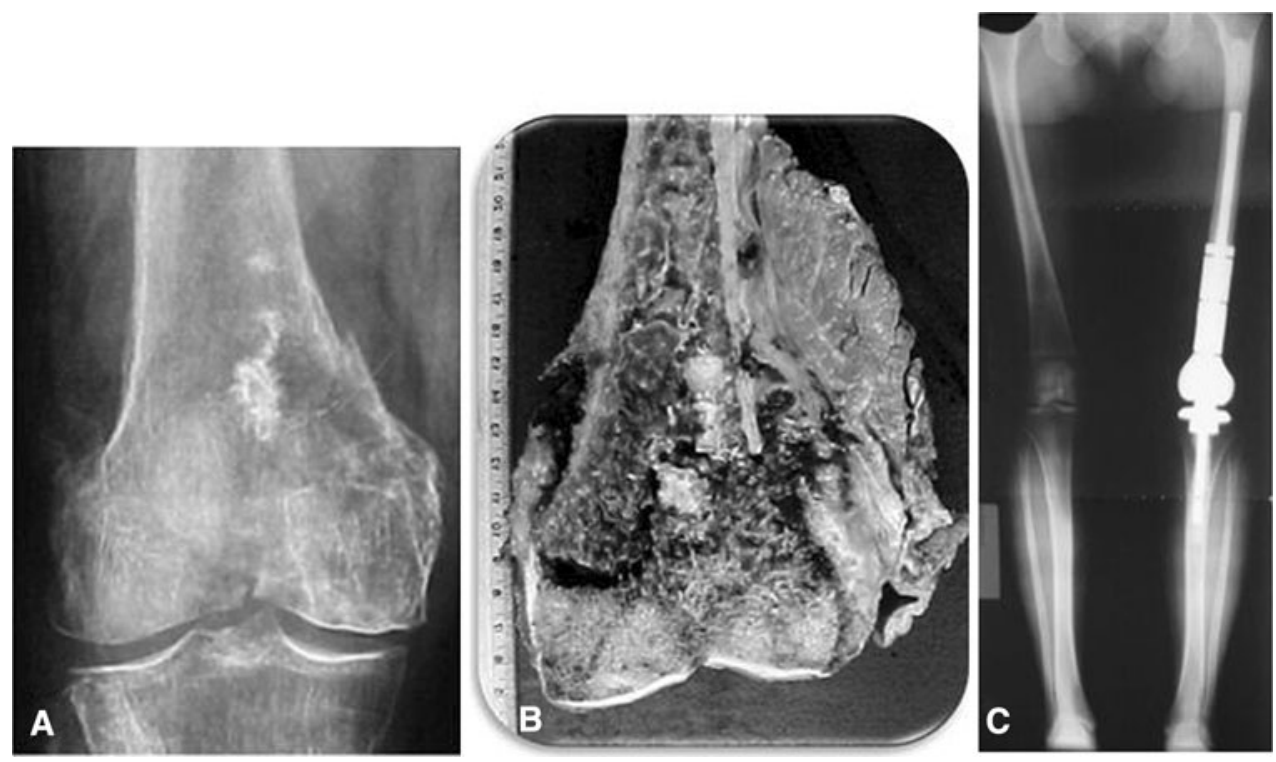

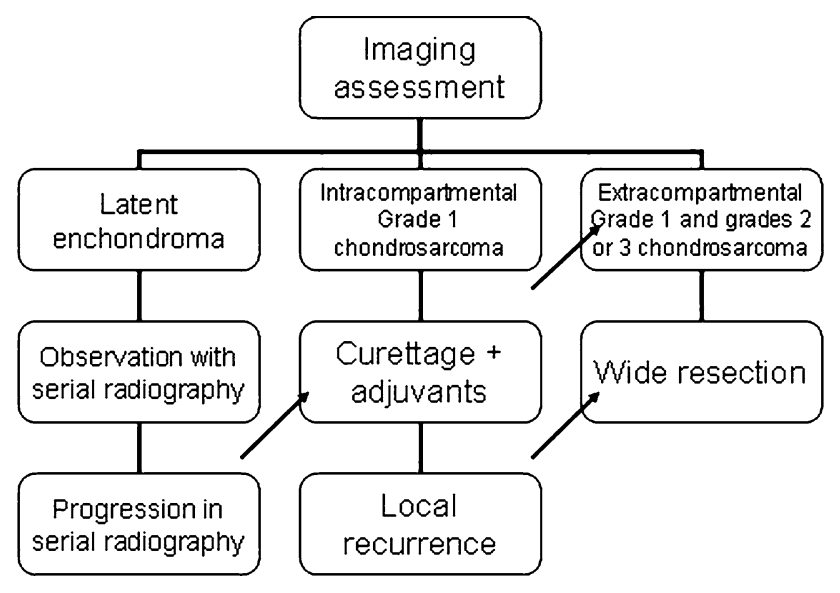

Fig. 5 The algorithm for primary central chondral bone lesion treatment is shown. sequence of diagnostic or therapeutic procedures in each clinical situation (Fig. 5).

\section{Discussion}

Primary central cartilaginous bone lesions present a challenge in diagnosis and treatment due to the wide biologic behavior spectrum of these tumors. The controversy resides in the fact that pathologic and radiographic grading of these tumors is not fully reliable [16]. Distinguishing an active enchondroma from a Grade 1 chondrosarcoma is often difficult, as imaging characteristics such as cortical thickening and endosteal scalloping may be present in both lesions. However, other radiographic signs, such as soft tissue mass, cortical expansion, thickening, and destruction, 
have been described and may indicate a Grade 2 chondrosarcoma [16]. Although these are subjective parameters, we believe that meticulous analysis of history, clinical signs, and radiographic characteristics can diminish the misjudgment of the chondrosarcoma grade to a comfortable level. In addition to the difficulty in diagnosis, there is also the issue of which surgical margin is adequate for each grade. There is no consensus about the adequate surgical margins for low grade tumors. Almost all authors agree that in grade 2 and 3 chondrosarcomas, a wide resection is mandatory. Grade 1 extracompartmental chondrosarcomas are also generally treated with wide resection. However, there is some controversy in Grade 1 intralesional chondrosarcomas. Streitbürger et al. recently published data that suggest that intralesional resection and cementation did not worsen either the local recurrence or the survival rate [17] and this finding was corroborated by Donati et al. [2]. We therefore compared patients with Grade 1 and Grade 2 primary central chondrosarcomas to (1) determine whether there is any difference in survival rates and (2) local recurrence rates; (3) determine whether there is any association of histological grade with clinical and demographic characteristics; and (4) to establish a treatment algorithm of central chondral bone lesions on the associations.

There are important limitations to our study. First, the study includes patients spanning 20 years, with different surgical teams and different pathologists. Thus, a possible bias would be the learning curve of surgeons and pathologists, working in a still controversial area of study, where no definitive evidence is available yet. Second, no patients were seen in followup for this study and the data were obtained from charts; and, in some cases, patients were lost for followup (there were no missing data). Nevertheless, according to the data presented here, we believe the algorithm described is adequate for managing central chondral bone lesions. Third, although we found a higher recurrence rate than the recent literature, we share the same difficulties as other orthopaedic oncologists regarding the management of chondrosarcomas. Therefore, larger, multicenter randomized prospective studies are needed to establish valid guidelines for these tumors.

In high-grade chondrosarcomas, local recurrence undoubtedly compromises survival [13]. However, there is controversy when Grade 1 chondrosarcomas are considered. There are studies suggesting that recurrences in Grade 1 axial skeleton chondrosarcomas do compromise survival [14]. However, other authors did not reach the same conclusion [6]. Schwab et al. observed a negative impact of local recurrence in survival in Grade 1 chondrosarcomas [15]. In this study we did not find correlation between local recurrence and survival for Grade 1 chondrosarcomas, since none of the six local recurrence patients died. On the other hand, all three Grade 2 patients who died of the disease experienced local recurrence after the resection procedure. When considering both groups, local recurrence negatively influenced survival.

We believe in the importance of standardized management of specific diseases. Achieving this objective is extremely difficult, especially when dealing with rare tumors such as chondrosarcomas: as stated by a recent study, the treatment algorithms have not improved survival rates in these patients yet [10]. Algorithms are an objective way of dealing with specific situations, and this is the reason we decided to analyze algorithm for primary central chondral bone lesions we currently employ at our service. According to the data presented here, this algorithm was proven adequate to manage these tumors, since no recurrence or survival information added by this research would justify changes in the sequence of procedures in each case. Our algorithm does not include biopsy of the lesion. Imaging assessment is mainly based on conventional radiography (two incidences). CT scan (for shoulder and pelvis girdles), MRI (for soft tissue mass detection and evaluation), bone scintigraphy, and PET-CT are additional optional imaging exams that may help determine the aggressiveness of the lesion. Once the radiography is thoroughly examined, the tumor must be classified either as a latent enchondroma, a Grade 1 chondrosarcoma, or a Grade 2 or 3 chondrosarcoma. If the presumptive diagnosis is a latent enchondroma, the patient is only observed with serial radiography. If the lesion progresses, intralesional resection, electrocauterization and cement is indicated. Grading of the resected specimen is carried out and if a grade 2 or 3 chondrosarcoma is unexpectedly found, then immediate wide resection is mandatory. For lesions with radiographic characteristics of intracompartmental Grade 1 chondrosarcoma, intralesional resection followed by electrocauterization and cement is the choice of treatment. If the lesion recurs, then en bloc resection is indicated. When the radiography shows signs of additional aggressiveness, suggesting a Grade 2 or even 3 chondrosarcoma, then wide resection is promptly indicated.

Acknowledgments We thank Tomaz Puga Leivas for his contribution in the statistical analysis of this study.

\section{References}

1. Björnsson J, McLeod RA, Unni KK, Ilstrup DM, Pritchard DJ. Primary chondrosarcoma of long bones and limb girdles. Cancer. 1998;83:2105-2119.

2. Donati D, Colangeli S, Colangeli M, Di Bella C, Bertoni F. Surgical treatment of grade I central chondrosarcoma. Clin Orthop Relat Res. 2010;468:581-589.

3. Donati D, El Ghoneimy A, Bertoni F, Di Bella C, Mercuri M. Surgical treatment and outcome of conventional pelvic chondrosarcoma. J Bone Joint Surg Br. 2005;87:1527-1530. 
4. Enneking WF. A system of staging musculoskeletal neoplasms. Clin Orthop Relat Res. 1986;204:9-24.

5. Eriksson AI, Schiller A, Mankin HJ. The management of chondrosarcoma of bone. Clin Orthop Relat Res. 1980;153:44-66.

6. Etchebehere M, Camargo OP, Croci AT, Oliveira NRB, Oliveira CRCM, Baptista AM. O papel da biópsia percutânea prévia no diagnóstico histológico definitivo na suspeita de lesões cartilaginosas malignas do esqueleto [The role of percutaneous biopsy in the definitive histological diagnosis of suspected cartilaginous bone lesions prior to surgery]. Rev Bras Ortop. 1999;34:77-80.

7. Etchebehere M, de Camargo OP, Croci AT, Oliveira CRGCM, Baptista AM. Relationship between surgical procedure and outcome for patients with grade I chondrosarcomas. Clinics. 2005;60:121-126.

8. Evans HL, Ayala AG, Romsdahl MM. Prognostic factors in chondrosarcoma of bone: a clinicopathologic analysis with emphasis on histologic grading. Cancer. 1977;40:818-831.

9. Fiorenza F, Abudu A, Grimer RJ, Carter SR, Tillman RM, Ayoub K, Mangham DC, Davies AM. Risk factors for survival and local control in chondrosarcoma of bone. J Bone Joint Surg Br. 2002;84:93-99.

10. Giuffrida AY, Burgueno JE, Koniaris LG, Gutierrez JC, Duncan R, Scully SP. Chondrosarcoma in the United States (1973 to 2003): an analysis of 2890 cases from the SEER database. J Bone Joint Surg Am. 2009;91:1063-1072.
11. Healey JH, Lane JM. Chondrosarcoma. Clin Orthop Relat Res. 1986;204:119-129.

12. Lee FY, Mankin HJ, Fondren G, Gebhardt MC, Springfield DS, Rosenberg AE, Jennings LC. Chondrosarcoma of bone: an assessment of outcome. J Bone Joint Surg Am. 1999;81:326338.

13. Lichtenstein L, Jaffe HL. Chondrosarcoma of bone. Am J Pathol. 1943;19:553-589.

14. Pant R, Yasko AW, Lewis VO, Raymond K, Lin PP. Chondrosarcoma of the scapula: long-term oncologic outcome. Cancer. 2005; 104:149-158.

15. Schwab JH, Wenger D, Unni K, Sim FH. Does local recurrence impact survival in low-grade chondrosarcoma of the long bones? Clin Orthop Relat Res. 2007;462:175-180.

16. Skeletal Lesions Interobserver Correlation among Expert Diagnosticians (SLICED) Study Group. Reliability of histopathologic and radiologic grading of cartilaginous neoplasms in long bones. J Bone Joint Surg Am. 2007;89:2113-2123.

17. Streitbürger A, Ahrens H, Balke M, Buerger H, Winkelmann W, Gosheger G, Hardes J. Grade I chondrosarcoma of bone: the Munster Münster experience. J Cancer Res Clin Oncol. 2009; 135:543-550.

18. Unni KK, Dahlin DC. Dahlin's Bone Tumors: General Aspects and Data on 11,087 Cases. 5th ed. Philadelphia, PA: LippincottRaven; 1996:71-72. 